\title{
A Prophylactic Role for Creatine in Hypoxia?
}

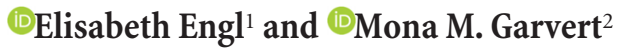 \\ ${ }^{1}$ Department of Neuroscience, Physiology and Pharmacology, University College London, London WC1E 6BT, United Kingdom, and ${ }^{2}$ Wellcome Trust \\ Centre for Neuroimaging, Institute of Neurology, University College London, London WC1N 3BG, United Kingdom \\ Review of Turner et al.
}

The brain requires $\sim 20 \%$ of the body's total energy. When the body is deprived of energy, such as during hypoxia and in pathologies involving an altered energy metabolism, the brain's function can be severely and irreversibly disrupted. However, humans routinely cope with transient hypoxia, for example when diving under water. How do our brains resist this temporary disruption in energy supply, and is it possible to harness these mechanisms for therapeutic use?

Much of the brain's energy is spent generating synaptic and action potentials and subsequently re-establishing the resting membrane potential by powering the $\mathrm{Na}^{+} / \mathrm{K}^{+}$-ATPase. To fuel these and other processes, the brain generates ATP. Under normoxic conditions, the predominant source of energy is the oxidative metabolism of glucose. During hypoxia, intracellular ATP levels fall and the $\mathrm{Na}^{+} / \mathrm{K}^{+}$-ATPase slows down. As a consequence, ionic gradients decrease. Neurons become depolarized and may either become more excitable or enter a depolarizing block of action potential production. To prevent neuronal damage by depolarization-evoked calcium entry, adenosine is released into the extracellular space, which in turn suppresses glu-

\footnotetext{
Received March 27, 2015; revised May 12, 2015; accepted May 13, 2015. This work was supported by the Wellcome Trust (4-year PhD studentship 097265/Z/11/Z to E.E. and 4-year PhD studentship 097267/Z/11/Z to M.M.G). We thank David Attwell, Renaud Jolivet, Erman Misirlisoy, and Peter Smittenaar for comments on the manuscript.

The authors declare no competing financial interests.

Correspondence should be addressed to Elisabeth Engl at the above address. E-mail: e.engl.11@ucl.ac.uk.

DOI:10.1523/JNEUROSCI.1195-15.2015

Copyright $\odot 2015$ the authors $\quad 0270-6474 / 15 / 359249-03 \$ 15.00 / 0$
}

tamate release from presynaptic terminals, reducing synaptic activity (Fowler, 1993). Brief hypoxia or even anoxia and the resulting adenosine release are reversible and do not lead to neuronal cell death (Miyamoto and Auer, 2000). However, the external $\mathrm{K}^{+}$ concentration jumps to $\sim 50 \mathrm{~mm}$ after a few minutes of anoxia, leading to a rapid depolarization of cellular membranes, the socalled "anoxic depolarization" (Hansen, 1985). A prolonged rise of extracellular glutamate concentration then triggers widespread neuronal cell death, after which anoxic damage becomes irreversible, leading to severe neuropsychological impairments.

Importantly, several reserve energy substrates can be used to replenish ATP without a need for oxygen, thus delaying the anoxia-induced alteration of ion gradients. In addition to glycogen, creatine is used as such an energy buffer in the brain. Creatine kinase catalyzes the dephosphorylation of phosphocreatine and the simultaneous phosphorylation of adenosine diphosphate (ADP) to ATP in the following reaction: $\mathrm{PCr}+\mathrm{ADP}+\mathrm{H}^{+} \leftrightarrow \mathrm{Cr}+\mathrm{ATP}$.

Physiological creatine and creatine kinase concentrations are not homogeneous throughout the brain, but instead reflect regional differences in energy demands (Pouwels and Frahm, 1998); indeed, creatine kinase level correlates with brain activity as measured with EEG (Sauter and Rudin, 1993). Furthermore, both in vitro and behavioral evidence suggest that creatine is used as an emergency energy substrate, at least for a short time. Rodent hippocampal slices with fourfold enriched phosphocreatine levels retain synaptic transmission in hypoxia three times as long as without extra phosphocreatine, and ATP levels take 90 instead of $30 \mathrm{~s}$ to decline (Lipton and Whittingham, 1982). While remaining constant at rest, the ratio of hippocampal phosphocreatine to ATP concentration declines significantly when glucose uptake is impaired under cognitive load, suggesting that phosphocreatine is converted to creatine to replenish ATP (Emmanuel et al., 2013).

Can artificially increasing phosphocreatine levels help conserve neuronal function in disorders of energy supply? Studies of the clinical benefits of creatine administration have been contradictory. In a mouse model of amyotrophic lateral sclerosis (ALS), a disease characterized in part by mitochondrial dysfunction and therefore impaired energy supply, oral creatine supplementation protected against neuronal loss and improved motor performance. Crucially, it also led to longer survival rates, from $144 \mathrm{~d}$ with an unsupplemented diet to $157 \mathrm{~d}$ with $1 \%$ creatine and $169 \mathrm{~d}$ with $2 \%$ creatine (Klivenyi et al., 1999). Klivenyi et al. (1999) hypothesized that creatine both acted as an energy buffer and prevented the mitochondrial transition pore from opening (O'Gorman et al., 1997), thereby preventing the mitochondrial swelling and cell death characteristic of ALS. In contrast, a comparable dose of creatine in ALS patients failed to improve motor behavior (Shefner et al., 2004). Similarly, in patients with Huntington's disease, which 
involves a defective energy metabolism and excitotoxicity, 2-year oral supplementation of creatine did not improve motor or cognitive function (Tabrizi et al., 2005).

Turner and colleagues (2015) tried to address these discrepancies by investigating whether prophylactic oral creatine intake prevented the detrimental effects of hypoxia on cognitive performance in humans. The authors tested corticomotor excitability by measuring motor-evoked potentials with TMS and assessed cognitive performance on a variety of tasks contained in a standard neuropsychological test battery under normoxic conditions and after 90 min of hypoxia. Participants $(n=15)$ underwent the test procedure twice. Seven days of placebo or oral creatine administration $(20 \mathrm{~g} / \mathrm{d})$, allocated according to a double-blind randomized procedure, were followed by a wash-out period of 5 weeks, after which the procedure was repeated with the other treatment (creatine or placebo, respectively). Using magnetic resonance spectroscopy, the authors demonstrated that creatine treatment increased creatine and phosphocreatine levels by $9.2 \%$ on average in a region of interest over the hand motor area, the same area used to determine corticomotor excitability.

Hypoxia was induced by having participants breathe air containing an inspired oxygen fraction of $10 \%$, or half the level in air at sea level. The arterial oxygen saturation fell by $19 \%$ as expected from the oxygen-hemoglobin dissociation curve (Turner et al., 2015, their Fig. 3), which is well below the clinical threshold for hypoxemia (Majumdar et al., 2011). To put that level of hypoxia into perspective, an inspired oxygen fraction of $10 \%$ approximately corresponds to air at the summit of Mount Whitney, at an altitude of around $4.4 \mathrm{~km}$.

Hypoxia impaired a wide range of neuropsychological measures, including alertness, executive function, and verbal memory. Creatine supplementation significantly reduced the detrimental effects of hypoxia on a factor the authors call "complex attention" (Turner et al., 2015,their Table 1 and Fig. 4). Turner and colleagues (2015) assessed complex attention by pooling performance errors across three cognitive tasks: a Stroop test, in which subjects concurrently processed the semantic and the visual features of a color word; a Shifting Attention test, in which subjects switched between task instructions; and a Continuous Performance test, in which subjects responded to target, but not distractor letters. The effect of creatine on composite memory, psychomotor speed, and the overall neurocognitive index were statistically inconclusive, even though all domains were adversely affected by hypoxia. Given the heterogeneous distribution of creatine in the brain, it is conceivable that these differences in behavior reflect differences in creatine sensitivity across networks engaged in these different tasks. Similarly, the differences in cognitive effects for hypoxia under placebo and under creatine might indicate that hypoxia and creatine differentially affect different cognitive domains.

It is worth noting that the authors tested participants on a battery comprising a large number of neurocognitive tests. Testing for independent effects would require corrections for multiple comparisons, which would have eliminated the statistically significant effect of creatine supplementation. However, in this case it is unlikely that all of the neurocognitive tests are truly independent. Several identical subscores were included in different composite cognitive domains scores, and different domains, such as psychomotor speed and reaction time, probably recruit similar cognitive processes. In consequence, corrections for multiple comparisons might be overly conservative. To tease apart the effects of creatine on specific cognitive processes, future studies using a similar cognitive test battery could first perform a dimensionality reduction to clearly identify independent cognitive domains.

Turner and colleagues (2015) hypothesize that the effect of creatine on cognitive performance might, at least in part, be due to creatine's energy-buffering capabilities. However, it is unlikely that creatine can supply energy for the entire 90 min of hypoxia. In the absence of ATP production, all the ATP in the rat brain will be consumed in $\sim 7 \mathrm{~s}$ (Allen et al., 2005). The concentration of free ATP in the rodent and human brain is $\sim 2.5 \mathrm{~mm}$, while the phosphocreatine concentration is $\sim 4.9 \mathrm{~mm}$ (Roth and Weiner, 1991). Thus, ATP produced from the physiological level of phosphocreatine should be able to lengthen the time until ATP is consumed by $\sim 12 \mathrm{~s}$ in rat (Allen et al., 2005), or $24 \mathrm{~s}$ in humans and nonhuman primates [because primate gray matter consumes only around half as much energy as rodent gray matter, or $0.2 \mathrm{~mm}$ ATP per second (Kennedy et al., 1978)]. Since the increase of creatine and phosphocreatine produced was $<1 \mathrm{~mm}$ (as shown in Turner et al. (2015), their Fig. 2A), any energy-buffering effect of the extra crea- tine must occur within a few seconds and should therefore be restricted to the first neuro-psychological test that participants performed. As Turner and colleagues (2015) do not indicate whether the order of the tests was randomized, it is not clear whether the selective effects of creatine on complex attention might be explained by it being the first test in the battery.

Any longer-lasting effect of creatine on cognitive performance would instead support the alternative hypothesis of Turner and colleagues (2015) of a substantial neuromodulatory role of creatine, possibly by directly enhancing the function of the $\mathrm{Na}^{+} / \mathrm{K}^{+}$-ATPase (Rambo et al., 2012). Creatine also directly stimulates glutamate uptake into synaptic vesicles (Xu et al., 1996).

Superficially, one might expect an increase of corticomotor excitability in hypoxia, altering the cortical excitation/inhibition balance with potentially detrimental consequences for cognitive performance. The neuromodulatory effects of creatine might then reverse these effects. Instead, Turner and colleagues (2015) saw no statistically significant effect of hypoxia on excitability in placebo, but an increased excitability with creatine (their Fig. 5A). However, the analyses in Figure 5 do not allow a direct comparison of changes in corticomotor excitability between placebo and creatine, as the amplitudes were separately normalized to the largest motor-evoked potential recorded in the placebo and creatine sessions, and so are not comparable across conditions.

In comparison to clinical studies using a similar dose of creatine that found no beneficial effects on cognitive performance, a crucial difference that could explain the efficacy of creatine supplementation in this study is that creatine was allowed to accumulate before energetic disruption. In the clinical studies by Shefner and colleagues (2004) and Tabrizi and colleagues (2005), creatine treatment was only started after ALS or Huntington's were already manifest. In contrast, creatine supplementation preceded disease onset in the ALS mouse model, where it was efficacious (Klivenyi et al., 1999). If a beneficial effect of creatine is successfully replicated, future clinical implementation could therefore focus on prophylactic, rather than post hoc, effects of creatine in the treatment of disorders involving changes in energy metabolism.

In conclusion, Turner and colleagues (2015) show that even relatively mild hypoxia impairs cognition, which might, at least in part, be recovered by oral creatine supplementation. This suggests that crea- 
tine helps to maintain normal cognitive function when energy metabolism is impaired, although the precise mechanism, as well as the reason that some cognitive processes are more affected than others, remain unexplained. As the energybuffering effects of added creatine are of brief duration (a few seconds), future mechanistic studies should focus on the neuromodulatory actions of creatine under prolonged hypoxia.

\section{References}

Allen NJ, Káradóttir R, Attwell D (2005) A preferential role for glycolysis in preventing the anoxic depolarization of rat hippocampal area CA1 pyramidal cells. J Neurosci 25:848-859. CrossRef Medline

Emmanuel Y, Cochlin LE, Tyler DJ, de Jager CA, Smith AD, Clarke K (2013) Human hippocampal energy metabolism is impaired during cognitive activity in a lipid infusion model of insulin resistance. Brain Behav 3:134-144. CrossRef Medline

Fowler JC (1993) Changes in extracellular adenosine levels and population spike amplitude during graded hypoxia in the rat hippocampal slice. Naunyn Schmiedebergs Arch Pharmacol 347:73-78. Medline

Hansen AJ (1985) Effect of anoxia on ion distribution in the brain. Physiol Rev 65:101-148. Medline

Kennedy C, Sakurada O, Shinohara M, Jehle J, Sokoloff L (1978) Local cerebral glucose utilization in the normal conscious macaque monkey. Ann Neurol 4:293-301. CrossRef Medline

Klivenyi P, Ferrante RJ, Matthews RT, Bogdanov MB, Klein AM, Andreassen OA, Mueller G, Wermer M, Kaddurah-Daouk R, Beal MF (1999) Neuroprotective effects of creatine in a transgenic animal model of amyotrophic lateral sclerosis. Nat Med 5:347-350. CrossRef Medline

Lipton P, Whittingham TS (1982) Reduced ATP concentration as a basis for synaptic transmission failure during hypoxia in the in vitro guinea-pig hippocampus. J Physiol 325:5165. CrossRef Medline

Majumdar SR, Eurich DT, Gamble JM, Senthilselvan A, Marrie TJ (2011) Oxygen saturations less than $92 \%$ are associated with major adverse events in outpatients with pneumonia: a population-based cohort study. Clin Infect Dis 52:325-331. CrossRef Medline

Miyamoto O, Auer RN (2000) Hypoxia, hyperoxia, ischemia, and brain necrosis. Neurology 54:362-371. CrossRef Medline

O'Gorman E, Beutner G, Dolder M, Koretsky AP, Brdiczka D, Wallimann T (1997) The role of creatine kinase in inhibition of mitochondrial permeability transition. FEBS Lett 414:253257. CrossRef Medline

Pouwels PJ, Frahm J (1998) Regional metabolite concentrations in human brain as determined by quantitative localized proton MRS. Magn Reson Med 39:53-60. CrossRef Medline

Rambo LM, Ribeiro LR, Schramm VG, Berch AM, Stamm DN, Della-Pace ID, Silva LF, Furian AF, Oliveira MS, Fighera MR, Royes LF (2012) Creatine increases hippocampal $\mathrm{Na}+, \mathrm{K}+$ ATPase activity via NMDA-calcineurin path- way. Brain Res Bull 88:553-559. CrossRef Medline

Roth K, Weiner MW (1991) Determination of cytosolic ADP and AMP concentrations and the free energy of ATP hydrolysis in human muscle and brain tissues with 31P NMR spectroscopy. Magn Reson Med 22:505-511. CrossRef Medline

Sauter A, Rudin M (1993) Determination of creatine kinase kinetic parameters in rat brain by NMR magnetization transfer. Correlation with brain function. J Biol Chem 268:1316613171. Medline

Shefner JM, Cudkowicz ME, Schoenfeld D, Con$\operatorname{rad}$ T, Taft J, Chilton M, Urbinelli L, Qureshi M, Zhang H, Pestronk A, Caress J, Donofrio P, Sorenson E, Bradley W, Lomen-Hoerth C, Pioro E, Rezania K, Ross M, Pascuzzi R, Heiman-Patterson T, et al. (2004) A clinical trial of creatine in ALS. Neurology 63:16561661. CrossRef Medline

Tabrizi SJ, Blamire AM, Manners DN, Rajagopalan B, Styles P, Schapira AH, Warner TT (2005) High-dose creatine therapy for Huntington disease: A 2-year clinical and MRS study. Neurology 64:1655-1656. CrossRef Medline

Turner CE, Byblow WD, Gant N (2015) Creatine supplementation enhances corticomotor excitability and cognitive performance during oxygen deprivation. J Neurosci 35:1773-1780. CrossRef Medline

Xu CJ, Klunk WE, Kanfer JN, Xiong Q, Miller G, Pettegrew JW (1996) Phosphocreatinedependent glutamate uptake by synaptic vesicles: a comparison with ATP-dependent glutamate uptake. J Biol Chem 271:1343513440. CrossRef Medline 